Cahiers d'études africaines

Parentés, plaisanteries et politique

\title{
Identités et relations de plaisanterie chez les Peuls de l'ouest du Burkina Faso
}

Youssouf DIALLO

\section{OpenEdition}

Journals

Édition électronique

URL : https://journals.openedition.org/etudesafricaines/15399

DOI : 10.4000/etudesafricaines.15399

ISSN : 1777-5353

Éditeur

Éditions de l'EHESS

\section{Édition imprimée}

Date de publication : 1 décembre 2006

Pagination : 779-794

ISBN : 978-2-7132-2129-3

ISSN : 0008-0055

Référence électronique

Youssouf DIALLO, «Identités et relations de plaisanterie chez les Peuls de l'ouest du Burkina Faso », Cahiers d'études africaines [En ligne], 184 | 2006, mis en ligne le 01 janvier 2008, consulté le 21

septembre 2021. URL : http://journals.openedition.org/etudesafricaines/15399; DOI : https://doi.org/ 10.4000/etudesafricaines.15399 
chercher : repérer : avancer

Cet article est disponible en ligne à l'adresse :

http://www.cairn.info/article.php?ID REVUE=CEA\&ID NUMPUBLIE=CEA 184\&ID ARTICLE=CEA 1840779

Identités et relations de plaisanterie chez les Peuls de l'ouest du Burkina Faso

\author{
par Youssouf DIALLO
}

\title{
Editions de l'EHESS | Cahiers d'études africaines
}

$2006 / 4-184$

ISSN 0008-0055 | ISBN 9782713221293 | pages 779 à 794

Pour citer cet article:

—Diallo Y., Identités et relations de plaisanterie chez les Peuls de l'ouest du Burkina Faso, Cahiers d'ét udes africaines 2006/4, 184, p. 779-794.

Distribution électronique Cairn pour Editions de l'EHESS .

(c) Editions de l'EHESS. Tous droits réservés pour tous pays.

La reproduction ou représentation de cet article, notamment par photocopie, n'est autorisée que dans les limites des conditions générales d'utilisation du site ou, le cas échéant, des conditions générales de la licence souscrite par votre établissement. Toute autre reproduction ou représentation, en tout ou partie, sous quelque forme et de quelque manière que ce soit, est interdite sauf accord préalable et écrit de l'éditeur, en dehors des cas prévus par la législation en vigueur en France. Il est précisé que son stockage dans une base de données est également interdit. 


\section{Identités et relations de plaisanterie chez les Peuls de l'ouest du Burkina Faso}

Cette contribution traite des relations de plaisanterie aux niveaux intra et interethnique, et décrit les aspects et caractéristiques majeurs de cette institution chez les pasteurs peuls (ou Foulbé) de l'ouest du Burkina Faso, d'une part, et entre les Peuls et leurs voisins sédentaires d'autre part. En effet, les relations de plaisanterie, résultant d'interactions sociales étroites, existent entre les Peuls et les membres de certains groupes socioprofessionnels, tels que la fraction mossi des Yarse (Fulfulde : Wangarbe, sing. Bangaro) ${ }^{1}$ et les forgerons, avec lesquels ils se disent liés par une alliance mythique, mais parfois aussi avec les membres de plusieurs ethnies avec lesquels les Peuls partagent simplement le même environnement spatial. Les plaisanteries interethniques, censées résulter d'une alliance ou simplement des relations de bon voisinage, sont d'une importance pratique pour les Peuls semi-nomades ou transhumants. De leur cohabitation avec les agriculteurs sédentaires se mettent en place des institutions socioculturelles qui favorisent leur intégration dans les sociétés sédentaires et limitent les conflits potentiels. Les formes d'alliance ou d'institution spécifique, comme les relations hôtes/étrangers, soulignent la nécessité des rapports fondés sur la complémentarité. De ce point de vue, les relations à plaisanterie jouent un rôle important dans le renforcement du processus d'intégration sociale des groupes mobiles, conduisant dans de nombreux cas à la résolution ou l'évitement des conflits entre les Peuls et les groupes d'accueil.

Dans l'exploration de l'univers et des formes de plaisanterie, nous commencerons tout d'abord par une description générale de cette institution sociale et ses caractéristiques à l'Ouest du Burkina Faso, un contexte marqué par une diversité ethnique, linguistique et culturelle. Il s'agira dans cette esquisse de situer dans un premier temps les relations peules/non-peules, analysées plus loin, dans une typologie générale de l'institution des plaisanteries. Ensuite, nous traiterons de la familiarité spécifique entre les Peuls,

1. Les lettres $\mathrm{F}$ et $\mathrm{D}$, marquées entre parenthèses, sont utilisées pour les termes fulfulde et dyula. 
d'une part, et entre les Peuls et leurs voisins d'autre part. Dans une section comparative, nous signalerons l'existence d'un type d'arrangement similaire, appelé hōlaare par les Peuls, qui met en évidence une relation structurelle marquée par la familiarité et la coopération entre personnes liées par un pacte (Fay 1995). Enfin, la discussion sur la familiarité est poursuivie et développée dans la section finale consacrée aux relations entre les Peuls et les forgerons.

\section{Pouvoir, identités et relations de plaisanterie}

Les relations de plaisanterie telles qu'on les observe en Afrique de l'Ouest ou ailleurs sont l'expression générale d'une forme d'amitié entre membres de sexe masculin et féminin d'une même famille élargie ou entre membres de certaines ethnies voisines et clans liés par des liens de parenté réels ou fictifs. Selon une version répandue à l'ouest du Burkina Faso sur les plaisanteries, et que l'on retrouve ailleurs en Afrique des savanes, si l'on se réfère à la littérature africaniste sur le sujet, la coutume des plaisanteries (sanankuya en dyula) entre groupes ethniques est très souvent le résultat d'une alliance ou d'un accord de paix entre deux ancêtres fondateurs qui auraient mis fin à un conflit les ayant opposé par un pacte de sang solennel ${ }^{2}$. Selon un mythème généralement répandu, deux ancêtres font saigner leur bras, boivent leur sang dilué dans de l'eau et décident de vivre en paix dorénavant (Paulme 1939; Tamari 1997). Ils décident de ne plus entrer en conflit, deviennent des partenaires rituels, coopèrent et rient des plaisanteries mutuelles. Le pacte de sang engage aussi leurs descendants respectifs, ce qui est important pour la continuité de l'institution de la plaisanterie. Le pacte inaugural, comparable à l'instauration du «pacte de sang » chez les Azande du Soudan (Evans-Pritchard 1962), confère un caractère sacré au contrat et parfois aussi une signification politique aux plaisanteries entre les parties contractantes. Il ne s'agit pas de faire une comparaison entre le pacte de sang chez les Azande et l'alliance à plaisanterie en Afrique de l'Ouest, mais de signaler simplement la différence entre les deux formes de pacte : chez les Azande les «frères de sang » peuvent échanger des filles comme épouses, alors que les partenaires rituels en Afrique de l'Ouest ne pratiquent pas, ou dans certaines conditions seulement, d'intermariages. Les relations Peuls/forgerons - sur lesquelles nous reviendrons - marquées par les plaisanteries, l'évitement et l'interdiction de rapports sexuels, constituent l'exemple classique de ce genre de «pacte de sang ». Ce pacte, dont la malédiction constitue un élément fondamental, possède une force morale. D'après une croyance, tout contrevenant est frappé par un malheur.

2. Voir aussi SisSAO (2002: 97-98) qui parle des conflits comme moments fondateurs des relations à plaisanterie. 
Dans les sociétés paysannes de l'ouest du Burkina Faso, on distingue habituellement les relations de plaisanterie de moindre importance (D. sanankuya missenw), limitées à de petites blagues et taquineries entre alliés ou membres de groupes voisins, et les plaisanteries intenses (D. sanankuya gwelenw). Les Peuls parlent de "tolo mangu $»^{3}$ pour caractériser des plaisanteries intenses. D'autres groupes mandés, tels que les Dyula, parlent quelquefois de plaisanteries «amères » ou acerbes par opposition aux plaisanteries douces ${ }^{4}$. Les plaisanteries amères émergent toujours dans des circonstances singulières. La distinction entre les deux formes de plaisanterie n'est pas fortuite dans la mesure où les plaisanteries amères entre deux alliés sont des ironies à la fois intenses et mordantes sans que l'un des deux partenaires ne se fâche. Toutefois, les plaisanteries acerbes et douces se distinguent les unes des autres quant aux circonstances de leur émergence et dans leur rapport au sacré. La différence entre les deux types de relations de plaisanterie n'est donc pas seulement de degré. Les croyances déterminent l'intensité et le sérieux avec lesquels les acteurs sociaux envisagent les plaisanteries considérées comme «vraies ». Plus une alliance est considérée comme sacrée, plus lourdes sont les obligations et acerbes les plaisanteries entre les alliés. Les plaisanteries intenses et acerbes, comme celles qui existent entre les Peuls et les forgerons, dans lesquelles on reconnaît une forme d'attachement aux systèmes de pensée en Afrique de l'Ouest, sont généralement le résultat d'une alliance sacrée, comme pour l'exemple mentionné ci-dessus. Sur le plan matrimonial, par exemple, les intermariages entre « vrais » partenaires rituels sont strictement interdits. La transgression des règles et tabous sexuels peut avoir de graves conséquences marquées par des sanctions occultes pouvant entraîner soit la malchance dans toute entreprise future soit la mort du partenaire fautif. C'est pourquoi la transgression des règles fixées dans le cadre des alliances à plaisanterie nécessite toujours une réparation sous forme de paiement de compensation ou sous forme de sacrifice expiatoire. L'appropriation d'objets appartenant à l'allié à plaisanterie est une autre caractéristique des relations à plaisanterie «vraies ». Dans des occasions aussi sérieuses que la levée du corps d'un partenaire à plaisanterie, un individu présent à la cérémonie peut se permettre de taquiner ses alliés à plaisanterie en deuil. Chez les groupes komono de l'ouest du Burkina Faso, un allié à plaisanterie peut même faire irruption dans une famille plongée dans le deuil, se saisir d'une chèvre ou d'un mouton. Un allié à plaisanterie a également droit à certaines parties d'un animal égorgé à l'occasion de funérailles.

3. «Tolo» (« jeu») et «mangu» (« grand ») signifient littéralement « grosse plaisanterie ». mangu ne doit pas être confondu avec le mangu entre les Dogon et les Bozo (Griaule 1948).

4. Voir aussi Launay (1977) sur les plaisanteries chez les Dyula du nord de la Côte-d'Ivoire. 
La plupart de nos interlocuteurs peuls et paysans s'accordent pour dire que l'interdiction d'alliance matrimoniale entre partenaires rituels est due au fait que les histoires de femmes sont des sujets sensibles entraînant souvent des conflits entre groupes ou individus. Permettre les intermariages mettrait en danger une alliance qui est pacifique par définition. Chez les Dogossié, la gifle infligée au partenaire fautif est un exutoire censé résoudre des démêlés provoqués par des histoires de femmes entre alliés à plaisanterie. Une deuxième gifle, infligée sous l'empire de la colère, est considérée comme un signe de malédiction qui nécessite une réparation par le don d'un animal.

Dans la façon dont les acteurs sociaux conçoivent et utilisent les plaisanteries, il apparaît que ce type de relations, né du souci de l'évitement des conflits, est une forme de familiarité et d'amitié entre plusieurs catégories de personnes. Au Burkina Faso, ces formes de familiarité mettent en relations des acteurs nombreux et variés. Ainsi, des relations de plaisanterie existent-elles entre membres de groupes ethniques différents, entre anciens occupants et nouveaux arrivants, entre grands-parents (D. mooke) et petitsfils (D. moodenw), entre cousins croisés (D. belendenw), entre oncles et neveux, ce dernier type étant une des expressions des relations avunculaires assez importantes chez les groupes de culture et langue voltaïques, en particulier les Dogossié, les Komono et les Sénoufo. Les plaisanteries, qui sont le résultat d'une alliance, existent entre castes et membres de plusieurs ethnies ou clans ou lignages, l'exemple typique étant l'alliance entre les forgerons (D. пити) et les griots (D. jeli) et certains groupes ethniques. Les relations nouées avec les castes sont de type symétrique, c'est-à-dire marquées par les plaisanteries et l'évitement, si l'on se réfère à la distinction de Radcliffe-Brown (1940).

Quant aux relations de plaisanterie entre autochtones (D. dugulewn) et nouveaux arrivants (D. lassiguidewn), elles existent surtout entre les Komono et les Dogossié de la région de Mangodara dans le sud-ouest du Burkina Faso, deux groupes ayant des traits communs, mais qui se distinguent les uns des autres par l'origine. Les traditions orales font venir les Dogossié du Nord du Ghana actuel, tandis que les groupes constitutifs de l'ensemble komono - hyperonyme dyula — seraient originaires de Kong ou passés par ce centre politique. Les traditions orales des Komono et des Dogossié font également état d'un conflit auquel les deux groupes auraient mis fin en concluant un «pacte de sang », dont les plaisanteries constituent actuellement un des aspects majeurs. Nos interlocuteurs dogossié racontent qu'à leur arrivée dans la région, les envahisseurs komono se seraient imposés par la force aux autochtones dogossié en s'appropriant les instruments d'extraction de l'or. Après une tentative de résistance, les Dogossié auraient fui pour aller se réfugier dans des grottes, avant de conclure un pacte avec les Komono. Ils utilisent aujourd'hui le terme konkaibe pour plaisanter avec leurs voisins komono qu'ils appellent les «intrus ». Les Komono, quant à 
eux, désignent les Dogossié «gens du trou » en référence à leur couardise. Cette forme de plaisanterie est la représentation de rapports de force passés entre groupes d'une même unité politique.

\section{Emblèmes nominatifs et systèmes d'équivalence}

La mention des relations entre Komono et Dogossié n'a pas simplement une valeur mythique dans l'étude des formes de plaisanterie. L'examen des aspects historiques du phénomène des plaisanteries dans ses moments constitutifs révèle que ce type de relations constitue le modèle restreint d'interactions sociales larges et relativement anciennes où se reflète la représentation de rapports de force passés entre groupes dominants et groupes dominés dans les formations étatiques précoloniales ouest-africaines. Les relations de clientèle Peuls/Bambara dans le cadre du royaume de Ségou, de même que les relations entre les chefs wattara/ouattara de l'ancien État de Kong et les groupes assujettis sont typiques de ce genre de relations entre dominants et dominés.

À Ségou, le roi bambara, qui se distinguait par la possession de bétail et d'esclaves, imposait des corvées, des tributs et la réquisition des groupes professionnels. Être Peul n'avait de sens que dans le cadre de ces relations de clientèle, car les pasteurs peuls devaient apporter leur contribution au rendement des troupeaux du clan royal (Monteil 1977 : 324). Pour les Bambara, un Peul doit toujours se rattacher à un maître au service de qui il garde le bétail, sans quoi les Bambara qualifient le Peul de «futile». Les relations Peuls/Bambara, qui illustrent les rapports de force passés et les familiarités entre les deux groupes, ne sont certes pas le résultat d'une alliance au sens où ces relations sont ritualisées à l'instar des relations Peuls/ forgerons, mais elles font partie des plaisanteries entre les deux groupes.

Les relations entre les dirigeants de l'ancien État de Kong et les groupes dominés offrent un autre exemple de ce type de liens entre pouvoir, identité et plaisanterie. Il ne s'agit pas ici de revenir sur l'histoire déjà connue de Kong (en Côte-d'Ivoire actuelle), mais simplement de rappeler que cet État est fondé au $\mathrm{XVIII}^{\mathrm{e}}$ siècle par des marchands dyula islamisés parvenus à sécuriser les voies commerciales reliant la zone pré-forestière à la vallée du Niger avec l'aide de paysans-soldats locaux (sonangui). Seku Ouattara, le fondateur de la dynastie, impose alors la domination dyula sur les Sénoufo de Kong et ses environs, tandis que ses successeurs se chargeront d'établir leur emprise sur toute la région située au nord de Kong, et notamment sur les groupes de l'ouest de l'actuel Burkina Faso (région de Bobo-Dioulasso). Cependant, les conquêtes dyula, effectuées loin de la base de Kong, n'étaient pas à proprement parler des annexions ou des contrôles territoriaux, mais une forme de suprématie politique par laquelle les groupes extérieurs étaient maintenus sous tutelle. La diffusion de l'emblème nominatif Ouattara fait 
partie de ce type de pouvoir de tutelle dont l'analyse des modalités d'exercice met en évidence les rapports entre l'identité ethnique ou clanique et l'institution des plaisanteries.

Les emblèmes nominatifs ou labels patronymiques sont des critères essentiels qui permettent, dans le cadre des relations à plaisanterie, le repérage au premier abord par un acteur social d'un allié. Ces emblèmes étaient d'abord des symboles statutaires ou des devises de clans utilisés par les groupes dirigeants des systèmes politiques précoloniaux et qui vont par la suite jouer un rôle important dans le processus d'intégration des étrangers, des dominés ou des captifs. La tendance à incorporer plus ou moins rapidement les fugitifs ou les captifs de guerre dans les États est une des caractéristiques des systèmes politiques africains anciens. En effet, les captifs et les dominés pouvaient adopter librement ou se voir imposer systématiquement les patronymes des groupes dominants pour bien marquer leur appartenance et leur incorporation dans les unités politiques d'accueil. C'est l'une des raisons pour lesquelles on trouve aujourd'hui en Afrique de l'Ouest les mêmes patronymes dans des groupes ethniques différents et qui sont répandus dans toute la zone des savanes et même pré-forestière (Pageard 1958; Launay 1977), tandis que d'autres sont spécifiques d'une localité et portés par un seul groupe ethnique ou professionnel (Conrad \& Franck 1995).

Les relations de plaisanterie sont en partie l'expression des rapports identitaires. L'identité ethnique ou sociale elle-même découlait d'un rapport de force. Chez les Mossi, une façon de se réadapter consistait pour les bannis, les exclus ou les captifs à abandonner leur milieu et à venir se mettre au service du roi en prenant son nom (Izard 1985). D'anciens captifs peuls (bagaredemba), affranchis de la tutelle de leurs maîtres, faisaient également partie des éléments serviles venus se soumettre volontairement à l'autorité du Moogo Naaba. Les individus d'origine peule de même que les membres du sous-groupe des Silmi-Mossi — formé à la fois de gens issus de l'union entre Peuls et femmes mossi et de Mossi qui ont pris l'identité peule ont des relations de plaisanterie avec les membres des groupes socioprofessionnels. Aujourd'hui, les Peuls et assimilés font des plaisanteries et n'ont pas de relations matrimoniales avec les forgerons mossi (patronymes kindo, zonne), les commerçants yarse (patronymes sore, guiro) et maranse (patronyme maïga) et les griots, joueurs de tam-tam (bendere).

À Ségou, «Fula» (ou Peul) était une étiquette que le clan royal collait à tous les individus, libres ou serviles, et groupes d'individus qui avaient pour activité principale la surveillance de troupeaux bovins (Monteil 1977 : 324). Les chefs de guerre dyula de Kong ont de la même façon donné ou imposé le nom Ouattara aux groupes morcelés et sans unité politique de l'Ouest de l'actuel Burkina Faso, soumis à leur autorité. Un interprète dyula a bien souligné, lors de la Table ronde sur les origines de Kong, organisée en 1977 par l'Université d'Abidjan, le caractère systématique de ce type d'assignation identitaire : 
«L'exercice du pouvoir des Ouattara, quand ils allaient quelque part, quand ils régnaient dans une région ou quand ils partaient en guerre contre un pays s'ils arrivaient à les battre, ce pouvoir ils pouvaient le confier à quelqu'un d'autre qui n'était pas Ouattara, une fois qu'ils lui avaient donné le pouvoir celui-ci portait le nom Ouattara » (1977: 164).

Dans la région de Mangodara, les Dogossié-fing de patronyme ouattara, un groupe d'anciens chasseurs peuls itinérants, devenus les sujets de Bamba, chef de guerre influent de Kong, et appelés pour cela Bamba-jon (esclaves ou sujets de Bamba), ont ainsi pris le nom Ouattara (Diallo 2004). À l'heure actuelle, les Bamba-jon ont pour alliés à plaisanterie les Kombe-jon (sujets de Kombe), membres d'une autre ancienne fraction armée de Kong. Un des aspects de cette spécificité, liée à cette institution, est que les relations de plaisanterie permettent aux Bamba-jon Dogossié qui se rendent à Kong d'avoir droit au logement chez les Kombe-jon, leurs correspondants ${ }^{5}$.

L'assignation identitaire ou l'usage politique qui a été fait de certains patronymes les ont privé de toute signification ethnique. Aujourd'hui, il y a des Ouattara dans l'ouest du Burkina Faso qui ne sont pas des Dyula et qui ne sauraient prétendre être issus directement de Kong, de même qu'il y a des Kulibali — leurs alliés à plaisanterie — qui ne sont pas des Bambaras et qui ne sont pas issus directement de Ségou. Des individus, qui portent le même patronyme, peuvent toutefois avoir un ou plusieurs alliés à plaisanterie dans d'autres groupes voisins ou éloignés. Par les relations d'équivalence entre labels patronymiques, les membres des castes ou de groupes ethniques alliés plaisantent et entretiennent des réseaux d'alliance. Ils coopèrent dans le cadre de la réciprocité et ont l'obligation de s'assister mutuellement par des échanges de services. Les équivalences et les homologies sont des indices de plaisanterie. En effet, le système codifié d'équivalences patronymiques, dont la familiarité est l'une des formes d'expression, constitue une sorte de grammaire des relations de plaisanterie, dont la connaissance et l'utilisation permettent de prévoir les attitudes et comportements d'alliés à plaisanterie, et cela indépendamment du contexte rural ou urbain. Si des patronymes considérés comme homologues entretiennent du fait de leur homologie des relations de plaisanterie, il reste que dans les relations entre groupes, il est toujours difficile de formuler des lois générales en raison de la diversité des facteurs environnementaux et historiques et en raison aussi de la diversité des conditions de vie humaine. Entre les équivalences et homologies d'une part, et les plaisanteries d'autre part, il n'y a donc pas nécessairement de relations de causes à effets; ces trois termes constituent des variables des grilles identitaires. Le tableau 1 (ci-après) est une présentation synoptique de ce type de correspondance envisageable entre noms claniques ou patronymiques distincts et alliés à plaisanterie dans

5. Entretiens avec Bamori Ouattara, chef de village, et un groupe de notables de Torandougou (Burkina Faso). Torandougou, 22 septembre 2000 et 20 novembre 2001. 
un contexte donné. La flèche signifie que tous les Ouattara du sud-ouest du Burkina Faso, indépendamment de leur appartenance ethnique (dyula, sénoufo ou komono), ont pour alliés à plaisanterie tous les Kulibali, Bamba, Koné, Diara, Kamara et, plus récemment, les Peuls de patronyme sidibé. Partout où un Ouattara et un Kulibali/Coulibaly ou un Diara se rencontrent, ils plaisantent.

Les Ouattara et leurs alliés à plaisanterie (y compris les Sidibe)

\begin{tabular}{lll} 
Ouattara & $\leftrightarrow$ & Kulubali \\
Ouattara & $\leftrightarrow$ & Koné \\
Ouattara & $\leftrightarrow$ & Bamba \\
Ouattara & $\leftrightarrow$ & Kamara \\
Ouattara & $\leftrightarrow$ & Diara \\
\hline Ouattara & $\leftrightarrow$ & Sidibe \\
\hline
\end{tabular}

\section{Relations d'équivalence et de plaisanterie chez les Peuls}

Les moments constitutifs des plaisanteries et les aspects historiques du phénomène, qui mettent en évidence les relations entre pouvoir et identité, viennent de faire l'objet d'une description. Les relations d'équivalence, de pouvoir, de propriété et d'identité ne sont pas observables uniquement dans les groupes et sociétés sédentaires dont nous venons de parler. On retrouve les mêmes caractéristiques chez les Peuls semi-nomades ou transhumants de l'ouest du Burkina Faso. Rappelons que l'ensemble ethnique peul est composé de quatre clans principaux : Diallo, Diakite, Sidibe et Sangare. Les couples Diallo/Diakite et Sidibe/Sangare, qui constituent le noyau mythique de la fulanité, sont en relations d'équivalence. Ces quatre clans aînés comprennent plusieurs sous-clans ou groupes patronymiques liés entre eux par des relations de plaisanterie dites dendiraagu, terme fulfulde utilisé pour désigner également les relations familières entre cousins croisés. En effet, les Peuls désignent les clans alliés et les cousins croisés indistinctement par le terme dendiraabe (sing. dendo), la plaisanterie elle-même prenant le nom de tolo (littéralement, jeu). Dans le premier couple, pour ne citer que cet exemple, H. Labouret rapporte que c'est dans une circonstance particulière qu'un Diallo et un Diakite ont noué une alliance. Au cours d'une fuite consécutive à une défaite, Diallo réanime Diakite, son compagnon défaillant, en lui faisant manger une partie de sa propre chair (Labouret 1929 : 252). Outre les couples initiaux que nous venons de mentionner, Diallo/Diko, Diakite/Ba et Sidibe/Sangare sont également homologues et entretiennent $\mathrm{du}$ fait de leur homologie des relations de plaisanterie (dendiraagu) et de confiance (hölaare). 


\section{Le hōlaare}

Un modèle classique de familiarité intense et de relations complémentaires décrit dans l'ethnographie ouest-africaine est l'alliance mangu qui lie les Dogons aux pêcheurs bozo de la boucle du Niger (Griaule 1948). Il semble que cette forme d'alliance ne soit pas spécifique de la zone, puisqu'une forme d'arrangement institutionnelle similaire, appelée hōlaare en langue peule, marquée par la familiarité, la coopération et l'entraide, existe entre les Peuls pasteurs de la zone et leurs voisins. On doit à Claude Fay (1995) une excellente analyse de l'arrangement hölaare entre les Peuls et les Bozo, comparable à l'alliance mangu entre ces derniers et les Dogon. Les échanges de railleries entre les Peuls et les Bozo ne représentent en réalité qu'une des facettes du système complexe qu'est le hōlaare ${ }^{6}$, vocable par lequel nos interlocuteurs peuls entendent une relation de confiance absolue qui peut exister entre membres de plusieurs lignages ou groupes peuls. Le hōlaare est aussi une alliance interethnique qui revêt deux aspects majeurs : un système d'articulation et de complémentarité entre groupes professionnels qui partagent un même espace écologique et une fonction d'intégration et de maintien de la paix (Fay 1995: 450).

L'alliance hōlaare, dont nous ignorons les conditions d'instauration dans les milieux peuls du Burkina Faso, régit également les relations intraethniques et a pour but le renforcement de la cohésion des sous-groupes peuls. Elle ne se confond pas avec le dendiraagu. Les clans qui se considèrent comme des amis entretiennent des relations de confiance fondées sur la coopération et l'évitement de conflits. Selon un de nos interlocuteurs, des rivalités ne doivent pas naître et opposer des clans peuls engagés dans cette forme d'alliance marquée par la confiance et la plaisanterie. Le hōlaare, souligne-t-il, est exclusif de l'échange matrimonial, alors que le dendiraagu ne l'est pas. Les Sidibe et les Bari de l'ancienne chefferie de Barani, par exemple, sont liés par le hōlaare. Lorsqu'un Sidibe rencontre un Bari, ils peuvent plaisanter. Un Sidibe en visite chez un Bari peut se permettre de voler les objets de ce dernier.

On pourrait ainsi multiplier les exemples de hōlaare au sein du groupe peul. Mais revenons aux relations dites dendiraagu pour signaler que les Diallo, les Diko et les Ba plaisantent intensément. Ainsi, un allié ba dirat-il d'un partenaire diallo qu'il est un mangeur de haricots, aliments peu prisés chez les Peuls. On dit aussi qu'un Diko de passage dans un endroit peut s'installer chez un Diallo même si le maître du lieu n'est pas là. Il peut y faire tout ce qu'il veut avant de continuer son chemin. Les Toroobe (patronyme tal), originaires du Fkta Toro dans le Sénégal actuel, sont les alliés à plaisanterie des Bingaabe habituellement classés dans le clan des Sangare. Les Sidibe ont pour alliés les Bari, tandis que les Baabe (patronyme

6. Hōlaare vient de hōla, qui signifie faire confiance à quelqu'un. Selon TAYLOR (1932: 84), hōlaare signifie aussi la paix, la sécurité ou la situation de paix. 
ba) plaisantent avec les Wolarbe (patronyme boli). Un des aspects du dendiraagu peul porte, outre les échanges de propos injurieux, sur la propriété et notamment le droit réciproque de commettre des larcins. Ainsi un Sidibe peut-il s'emparer de tout ce qu'il trouve chez un Bari, y compris parfois ses biens les plus précieux qui, alors, ne sont remis au propriétaire que contre paiement d'une compensation. La relation étant symétrique, un Bari peut également s'approprier le cheval ou la vache d'un Sidibe.

Clans peuls en relations de plaisanterie et de hōlaare (ci-dessous Sidibe et Bari)

\begin{tabular}{lll} 
Diallo & $\leftrightarrow$ & Diko \\
Diallo & $\leftrightarrow$ & Ba \\
Diallo & $\leftrightarrow$ & Diakite \\
Ba & $\leftrightarrow$ & Boli \\
\hline Sidibe & $\leftrightarrow$ & Bari \\
\hline
\end{tabular}

Compte tenu de la diversité des contextes ethniques et du fait de la migration des Peuls, les relations de plaisanterie ne varient pas seulement de nom patronymique à nom patronymique, mais aussi de groupe à groupe. Il existe en effet, sur le plan interethnique, des relations de plaisanterie entre les Peuls et plusieurs groupes non peuls, tels que les Bobo/Bwa, les forgerons et les commerçants mossi yarse, appelés Wangarbe (sing. Bangaro) par les Peuls.

\section{Les relations de plaisanterie peules/non-peules}

Le fait que les Peuls soient un groupe mobile explique l'existence de relations de plaisanterie entre eux et toute une série de populations sédentaires. De ce point de vue, leur position contraste avec celle des groupes et sociétés sédentaires du Burkina Faso qui n'ont souvent qu'un nombre très limité de partenaires à plaisanterie dans d'autres ethnies. Les Peuls semi-nomades ou transhumants doivent s'intégrer dans des contextes ethniques et culturels toujours nouveaux pour eux.

L'histoire et le voisinage sont les facteurs déterminants dans l'instauration des relations de plaisanterie entre les Peuls et les groupes sédentaires. De ce point de vue, une des formes les plus anciennes de plaisanterie interethnique est celle qui existe entre les Peuls, établis de longue date dans les communautés villageoises, et leurs voisins bobo et bwa. Nous avons déjà étudié ailleurs les moments constitutifs des relations entre pasteurs et sédentaires (Diallo 1997). Rappelons simplement que c'est au XIX siècle que les anciens nomades fondent dans la mouvance des jihad peuls, les chefferies de Barani et de Dokwi et réussissent à imposer leur domination sur les communautés villageoises où ils conduisent une politique de prédation au détriment des paysans bobo et bwa. Dans les communautés villageoises 
bobo et bwa, dont l'histoire est marquée par l'absorption constante d'éléments étrangers, les classifications ethniques correspondent aux classifications professionnelles. Jean Cremer (1924:21) signale ainsi l'existence d'une fraternité mythique entre le Bwa (agriculteur), le forgeron, le Peul (éleveur) et le Dyula (commerçant), à la base de laquelle certains voient l'origine des relations entre le Peul, le Bwa et surtout le forgeron. A. Sissao (2002 : 91), qui note que les guerres et conflits du passé ne sont pas seulement sources de division entre groupes ethniques, mentionne dans le cas des Bobo et des Peuls une version des relations à plaisanterie entre les deux groupes. En tout état de cause, les relations de voisinage historiques et le mythe contribuent à expliquer aujourd'hui les familiarités entre les Peuls, d'une part, et les Bwa et les Bobo, d'autre part, qui se taquinent à chaque occasion. Les partenaires bobo/bwa et peuls rient des plaisanteries sur le caractère moral (trompeur, stupide), le statut social (esclave) ou le mode de consommation (buveur de bière de mil). En référence à l'histoire passée des razzias des cavaleries peules, le Peul appelle le Bobo son esclave ou le qualifie de stupide. En retour, le Peul entendra, partout où il passe, qu'il est un individu futile ou un vrai filou, un jugement qu'exprime aussi la comédie et proverbe dyula: "janfa juru, karabara juru », une formule que l'on peut rendre par l'expression française «homme de sac et de corde». La possibilité de plaisanteries grossières entre les Peuls et leurs partenaires n'est jamais exclue, plaisanteries qui peuvent porter sur des insultes obscènes ou macabres. Un jour, à Bobo-Dioulasso, nous avons été témoin d'une scène de plaisanterie lugubre entre un Peul et un Bobo: «Toi, le Bobo stupide qu'est-ce que tu fais là !», dit le Peul, et le Bobo de rétorquer : « C'est ton père qui gît au cimetière qui est stupide...»

Cette forme de plaisanterie amère ou les petites blagues, qui ont pour cadre aussi bien le milieu rural qu'urbain, concerne également les fonctionnaires ou les bureaucrates bobos et peuls. Dans les plaisanteries en milieu urbain, les différences statutaires marquées par l'autorité, la hiérarchie et autres barrières sociales disparaissent généralement. Les exemples de relations de plaisanterie que nous venons de citer n'impliquent pas forcément d'interdiction d'alliance matrimoniale, comparable à celle que l'on observe dans les relations Peuls/forgerons ou Peuls/Yarse.

\section{Les relations Peuls/forgerons}

L'alliance la plus sacrée est probablement celle qui existe entre les Peuls et les forgerons (baylo en Fulfulde). Il s'agit d'une relation ritualisée que l'on retrouve entre les Peuls et les griots (musiciens). Les relations Peuls/ forgerons, qui combinent proximité et distance, correspondent à l'opposition structurelle soulignée par Radcliffe-Brown (1940), qui implique familiarité et évitement (Pageard 1958: 136). Amselle (1996: 757), quant à lui, voit dans les relations entre Peuls et forgerons une illustration des relations de 
clientèle à la base desquelles se trouve l'échange de biens et de services. En effet, les règles de l'alliance entre Peuls et forgerons prescrivent l'entraide réciproque, l'évitement de conflit entre les membres des deux groupes, de même que l'interdiction d'alliance matrimoniale. Une personne qui fait couler le sang de son partenaire rituel doit payer une compensation pour cela. Les Peuls et les forgerons plaisantent beaucoup et en toute circonstance. À la mort d'un Peul, un forgeron peut opposer un « refus rituel ${ }^{7}$ à son enterrement en empêchant par tous les moyens la levée du corps, et ce jusqu'à ce que ses partenaires peuls lui offrent de l'argent. C'est au forgeron qu'incombe d'ailleurs le rôle de médiateur en cas de conflit.

L'origine de la familiarité et de l'évitement dans les relations entre Peuls et forgerons se trouve dans les mythes des Peuls. Selon une version répandue de ce mythe sur l'échange réciproque de services, mentionnée par Denise Paulme (1939 : 440-441), le forgeron des temps anciens ressemblait à un singe avec beaucoup de poils et une longue queue. Le forgeron devient l'ami du Peul qui l'invite en ville. Le forgeron accepte. Le Peul rase son ami et lui coupe la queue avant de l'emmener en ville. En retour, le forgeron circoncise le Peul. Pendant l'opération, le sang du forgeron et celui du Peul se mélangent. Ils jurent alors de ne plus se blesser. Leurs descendants entretiennent des relations de plaisanterie, et pratiquent l'évitement sexuel ${ }^{8}$.

Une autre version des relations de plaisanterie, recueillie par nos soins, fait remonter les relations entre Peuls et forgerons à l'époque de la division du travail et du transfert de bétail notamment. La duperie est considérée comme un trait caractéristique du Peul qui cherche, par ce moyen, à acquérir du bétail au détriment des populations sédentaires. Au Niger, on raconte que les Peuls ont volé les bœufs des Haoussas pour s'enrichir (Dupire 1970 : 433, 1996 : 36-37). Pour la région que nous considérons, A. Sissao (2002 : 92) rapporte que les plaisanteries entre les Peuls et les forgerons sont basées sur le fait qu' "un Peul éleveur a volé les bœufs des forgerons bobo ». D'après une légende similaire ${ }^{9}$, le Peul aurait acquis le bétail au détriment $\mathrm{du}$ forgeron qu'il a dupé, alors qu'ils étaient de bons amis. Le forgeron travaillait dur pour acquérir du bétail qu'il confia en gardiennage à son ami peul. Pour éviter la confusion de leurs propriétés respectives, le Peul proposa au forgeron d'utiliser des marques de propriété différente. Les animaux du forgeron porteront des feuilles vertes, alors que le Peul utilisera des feuilles sèches. Peu après, tous les bœufs portaient des feuilles sèches. Le Peul informa alors le forgeron de la perte de ses bêtes portant les feuilles vertes. Le forgeron s'avisa par la suite de la duperie dont il avait été victime. Les deux partenaires auraient ri de cette situation et décidé de maintenir des relations d'amitié et de coopération.

7. Nous empruntons cette formule à Moreau (1944).

8. CAMARA (1992: 84) donne une version similaire dans son livre sur la condition et le rôle des griots (jeli en dyula) dans la société malinké.

9. Racontée par un de nos interlocuteurs : entretien avec D. Sidibé, 12 novembre 1996, Tengréla. 
Dans l'étude des relations et des alliances à plaisanterie en Afrique de l'Ouest, les Peuls semi-nomades et transhumants occupent une place remarquable compte tenu de la diversité de leurs alliés à plaisanterie. L'étude du phénomène des plaisanteries dans ses moments constitutifs que sont l'alliance, les relations de parenté et de voisinage supposait que soient décrits et analysés les aspects non seulement actuels, mais aussi historiques des relations à plaisanterie. L'institution de la plaisanterie n'est pas simplement un phénomène ludique des ethnies, clans et castes en Afrique de l'Ouest. Pour saisir les relations à plaisanterie dans toutes leurs dimensions, il importe donc de les voir comme phénomène social total, au sens maussien de l'expression, et prendre en considération toute une série d'autres aspects qui se rapportent au rituel, pouvoir, identité, la propriété de même que les échanges de biens et de services entre groupes alliés. Les relations de plaisanterie en tant que forme d'amitié impliquent l'évitement des conflits. L'aspect, dont il n'a pas été question ici, concerne les fonctions de régulation des conflits et d'intégration des relations à plaisanterie. L'attention récente portée à cette institution au Burkina Faso, comme dans les pays voisins (Mali, Côted'Ivoire), est le reflet d'une préoccupation politique générale et légitime, préoccupation qu'expliquent les guerres civiles actuelles. Cependant, l'idée que les relations à plaisanterie puissent être utilisées comme mécanisme de prévention et de résolution des conflits est une hypothèse controversée. Les débats et controverses nourris par cette idée sont à la base de l'intérêt récent porté par les intellectuels et activistes africains aux relations à plaisanterie (Fouéré 2005). Sans vouloir participer à la controverse, on notera que l'utilisation des relations à plaisanterie comme mécanisme de prévention et de règlement des conflits est une hypothèse généreuse mais réductrice des causes des conflits actuels dans les États africains postcoloniaux. Les «nouvelles guerres » sont complexes ; les questions d'exclusion et de partage du pouvoir se mêlent aux revendications identitaires et à la compétition pour le contrôle des ressources. Il serait illusoire, voire naïf, d'espérer résoudre les questions éminemment complexes de la prévention et de la résolution des guerres civiles par les relations à plaisanterie.

Max-Planck-Institut für ethnologische Forschung, Halle/Saale. Institut für Ethnologie, Leipzig. 


\section{BIBLIOGRAPHIE}

AMSelle, J.-L.

1996 «L'étranger dans le monde manding et en Grèce ancienne : quelques points de comparaison », Cahiers d'Études africaines, XXXVI (4), 144 : 755-761.

CAMARA, S.

1992 [1969] Gens de la parole. Essai sur la condition et le rôle des griots dans la société malinké, Paris, Karthala-ACCT ; Conakry, SAEC.

Conrad, D. C. \& Frank, B. E. (eds.)

1995 Status and Identity in West Africa: Nyamakalaw of Mande, Bloomington, Indiana University.

CRemer, J.

1924 Les Bobo (la vie sociale), Paris, Paul Geuthner.

DiALlo, Y.

1997 Les Fulbe du Boobola, Cologne, Köppe.

2004 Les Peuls des espaces interstitiels. Pastoralisme, migration et identités (Burkina Faso, Côte d'Ivoire, Thèse d'habilitation, multigr., Leipzig, Université de Leipzig.

DUPIRE, M.

1970 Organisation sociale des Peul : étude d'ethnographie comparée, Paris, Plon.

1996 Peuls nomades: étude descriptive des Wodaabe du Sahel nigérien, Paris, Karthala.

EVANS-PRITCHARD, E. E.

1962 Essays in Social Anthropology, London, Faber \& Faber.

FAY, C.

1995 «Car nous ne faisons qu'un. Identités, équivalences, homologies au Maasina (Mali) », Cahiers des Sciences humaines, 31 (2) : 427-456.

FOUÉRÉ, M.-A.

2005 «Les métamorphoses des "relations à plaisanteries". Un nouvel enjeu politique dans la construction des États-nations », Cahiers d'Études africaines, XLV (2), $178: 389-430$.

Griaule, M.

1948 «L'alliance cathartique », Africa, 18 (4) : 242-258.

IZARD, M.

1985 Gens du pouvoir, gens de la terre: les institutions politiques de l'ancien royaume du Yatenga (bassin de la Haute-Volta blanche), Cambridge, Cambridge University Press; Paris, Éditions de la Maison des sciences de l'Homme.

LABOURET, H.

1929 «La parenté à plaisanteries en Afrique occidentale», Africa, $2:$ 244-254. 
LaUnAY, R.

1977 «Joking Slavery», Africa, 47 (4) : 413-422.

Monteil, C.

1977 Les Bambara du Ségou et du Kaarta: Étude historique, ethnographique et littéraire d'une peuplade du Soudan français, Paris, Maisonneuve et Larose.

Moreau, R. E.

1944 «Joking Relationships in Tanganyika », Africa, 14 (7) : 386-400.

PAGEARD, R.

1958 « Notes sur les rapports de "senankouya" au Soudan français particulièrement dans les cercles de Segu et de Macina», Bulletin de l'IFAN, 20 (1-2): 123-141.

Paulme, D.

1939 «Parenté à plaisanteries et alliance par le sang en Afrique occidentale », Africa, 12 (4) : 433-444.

RADCLIFFE-BRown, A. R.

1940 « On Joking Relationships », Africa, $13: 195-210$.

SissaO, A. J.

2002 Alliances et parentés à plaisanterie au Burkina Faso : mécanismes de fonctionnement et avenir, Ouagadougou, Sankofa \& Gurli Éditions.

TAMARI, T.

1997 Les castes de l'Afrique occidentale: artisans et musiciens endogames, Nanterre, Société d'ethnologie.

TAYLOR, F. W.

1932 A Fulani-English Dictionary, Oxford, The Clarendon Press.

Université NATIONALE DE CÔTE-D’IVOIRE

1977 Table ronde sur les origines de Kong, Abidjan, Université nationale de Côted'Ivoire, multigr.

\section{RÉSUMÉ}

L'article, qui porte sur les relations de plaisanterie chez les pasteurs peuls et leurs voisins sédentaires de l'ouest du Burkina Faso, décrit les aspects historiques et actuels de cette institution sociale. Il souligne l'importance et la variété des formes des plaisanteries interethniques qui tiennent à la dispersion des Peuls et à la nécessité pour eux de s'intégrer dans les sociétés sédentaires. Sont discutées, outre les équivalences, homologies et formes de familiarité au sein même du groupe peul, les relations de plaisanterie peules/non-peules de même que l'alliance entre Peuls et forgerons. 


\begin{abstract}
Identity and Joking Relationships among the Fulani in Western Burkina Faso. - The article deals with joking relationships between pastoral Fulani and their neighbours in western Burkina Faso, and describes historical as well as contemporary aspects of this social institution. It underlines the significance of inter-ethnic joking and its various forms in connection with the dispersal of Fulani and the necessity for them to integrate sedentary societies. Besides equivalences and homologies, forms of familiarity among the Fulani, and between them and non-Fulani groups as well as blacksmiths/Fulani joking-alliance are among the topics discussed.
\end{abstract}

Mots-clés/Keywords : Burkina Faso, Peuls, familiarités, identités, plaisanteries, sédentaires/Burkina Faso, Fulani, familiarity, identity, joking, sedentary. 